\section{Reconstruction of hit time and hit position of annihilation quanta in the J-PET detector using the Mahalanobis distance}

\author{
Neha Gupta Sharma, Michal Silarski, \\ Tomasz Bednarski, Piotr Białas, Eryk Czerwiński, \\ Aleksander Gajos, Marek Gorgol, Bożena Jasińska, \\ Daria Kamińska, Łukasz Kapłon, Grzegorz Korcyl, \\ Pawel Kowalski, Tomasz Kozik, \\ Wojciech Krzemień, Ewelina Kubicz, \\ Szymon Niedźwiecki, Marek Pałka, \\ Lech Raczyński, Zbigniew Rudy, \\ Oleksandr Rundel, Artur Stomski, Adam Strzelecki, \\ Anna Wieczorek, Wojciech Wiślicki, Marcin Zieliński, \\ Bożena Zgardzińska, Paweł Moskal
}

\begin{abstract}
The J-PET detector being developed at the Jagiellonian University is a positron emission tomograph composed of the long strips of polymer scintillators. At the same time, it is a detector system that will be used for studies of the decays of positronium atoms. The shape of photomultiplier signals depends on the hit time and hit position of the gamma quantum. In order to take advantage of this fact, a dedicated sampling front-end electronics that enables to sample signals in voltage domain with the time precision of about 20 ps and novel reconstruction method based on the comparison of examined signal with the model signals stored in the library has been developed. As a measure of the similarity, we use the Mahalanobis distance. The achievable position and time resolution depend on the number and values of the threshold levels at which the signal is sampled. A reconstruction method as well as preliminary results are presented and discussed.
\end{abstract}

Key words: J-PET detector $\bullet$ Mahalanobis distance $\bullet$ time resolution $\bullet$ threshold levels

N. G. Sharma ${ }^{\bowtie}$, T. Bednarski, P. Białas, E. Czerwiński, A. Gajos, D. Kamińska, G. Korcyl, T. Kozik, E. Kubicz, P. Moskal, Sz. Niedźwiecki, M. Pałka, Z. Rudy,

O. Rundel, M. Silarski, A. Słomski, A. Strzelecki, M. Zieliński

Faculty of Physics, Astronomy and Applied Computer Science, Jagiellonian University,

11 S. Łojasiewicza Str., 30-348, Kraków, Poland,

E-mail: pnp.neha@gmail.com

\section{Ł. Kapłon, A. Wieczorek}

Faculty of Physics, Astronomy and Applied Computer Science, Jagiellonian University,

11 S. Łojasiewicza Str., 30-348, Kraków, Poland and Institute of Metallurgy and Materials Science of the Polish Academy of Sciences,

25 W. Reymonta Str., 30-059, Kraków, Poland

M. Gorgol, B. Jasińska, B. Zgardzińska Department of Nuclear Methods, Institute of Physics, Maria Curie-Sklodowska University,

1 M. Curie-Sklodowskiej Sq., 20-031, Lublin, Poland

P. Kowalski, L. Raczyński, W. Wiślicki

Świerk Computing Centre,

National Centre for Nuclear Research,

7 Andrzeja Soltana Str., 05-400 Otwock/Świerk, Poland

W. Krzemień

High Energy Physics Division,

National Centre for Nuclear Research,

7 Andrzeja Soltana Str., 05-400 Otwock/Świerk, Poland

Received: 5 July 2015, Accepted: 28 August 2015

\section{Introduction}

A new concept of large acceptance the Jagiellonian positron emission tomograph (J-PET) based on the polymer scintillators [1-9] has been developed recently, which provides a solution for a whole-body positron emission tomograph (PET) imaging with an improved time of flight (TOF) resolution ${ }^{1}$, as it relies more on the precise time measurement than on energy resolution (like in traditional PETs) and the signals that we probe are very narrow few nanoseconds. To this end, a dedicated new electronics was developed, which enables signal sampling in voltage domain with a time precision of about 20 ps [13]. Moreover, we have been developing new methods of gamma quanta hit time and hit position reconstruction to fully exploit potential of the new tomograph $[11,14,15]$. One of the developed methods is based on the comparison of measured signals with respect to a library of synchronized model signals registered for a set of well-defined positions of scintillation points. The hit position is reconstructed as the one corresponding to the signal from the library that is most similar to the measured signal [11]. The measured signal can also be compared to the averaged model signals determined for each scintillation point that speeds up significantly the reconstruction [12]. The degree of similarity between the measured and the reference signals from the library can be

\footnotetext{
${ }^{1}$ In current tomographs, the best TOF resolution was achieved with $\mathrm{LSO}\left(\mathrm{Lu}_{2} \mathrm{SiO}_{2}\right)$ crystals and it is equal to about $400 \mathrm{ps}$ [10] and in our previous studies with the plastic scintillators, $\sim 290$ ps was achieved $[11,12]$.
} 
expressed by several metrics, e.g. the methods described in the articles $[11,12]$ use $\chi^{2}$ metric. In the present article, we discuss a method based on the Mahalanobis distance [16], which accounts for the correlation among the compared variables.

\section{Principle of time and position reconstruction using library of model signals}

The method was validated by using the experimental data collected by means of the double-strip J-PET prototype and ${ }^{22} \mathrm{Na}$ isotope as a source of annihilation gamma quanta. For noise suppression and selection of annihilation gamma quanta, a coincident registration of signals from both detectors was used. Pedestal correction was also implemented to all the collected signals. In order to construct the library only with the high-energy deposition events, only those signals were selected for which number of registered photoelectrons is greater than half of the number of photoelectrons corresponding to the Compton edge for 511-keV gamma quanta. Next, a two-dimensional plot of number of photoelectrons obtained from left- and right-sided photomultipliers connected to the same strip was bisected into four regions (region 1 , region 2 , region 3 , and region 4 ). An example for central position is shown in Fig. 1. Bisection was done in order to suppress the uncertainties arising due to the variation of time resolution as a function of number of registered photoelectrons. Then, for each bisected region, signals were synchronized by shifting their time scales in such a way that time of the gamma quantum hit inside the detector is the same for all events in the library. Finally, the shape of a model signal for each bisected region is determined by averaging pedestal corrected and synchronized signals ${ }^{2}$.

For reconstructing the hit-position of gamma quanta in the detector, the measured signals are compared with each model signal from the library. Signals can be represented as a vector of time values at defined threshold levels. In this article, we consider two threshold cases $(-60 \mathrm{mV}$ and $-120 \mathrm{mV})$ where $t_{\mathrm{L} 1}, t_{\mathrm{L} 2}$ and $t_{\mathrm{R} 1}, t_{\mathrm{R} 2}$ are time values on left and right signals (registered simultaneously at both ends of the scintillator) defined at $-60 \mathrm{mV}$ and $-120 \mathrm{mV}$ threshold levels, respectively:

$$
\vec{t}=\left[t_{\mathrm{L} 1}, t_{\mathrm{L} 2}, t_{\mathrm{R} 1}, t_{\mathrm{R} 2}\right] .
$$

The position and time of the examined signal is determined by minimizing the Mahalanobis distance (Eq. (2)) between this signal and the model signals stored in the library. For each

$$
M(z, \delta t, \text { region })=\sqrt{\begin{array}{l}
\vec{X}(\delta t, z, \text { region })] \\
\cdot[\operatorname{Cov}(z, \text { region })]^{-1} \\
\cdot \vec{X}(\delta t, z, \text { region })]
\end{array}}
$$

${ }^{2}$ For detailed descriptions of experimental setup and the

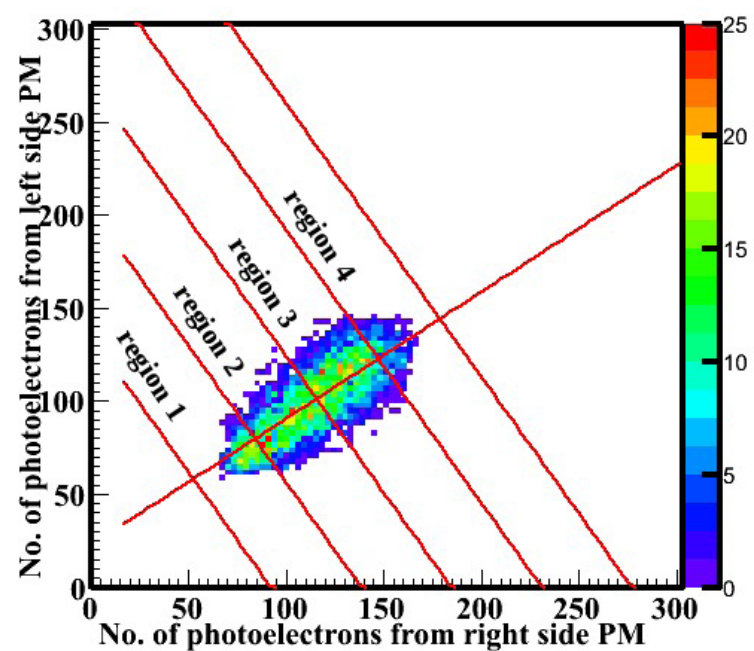

Fig. 1. Distribution of number of photoelectrons obtained from left- and right-sided photomultipliers connected to scintillator at central position.

$$
\begin{aligned}
& \operatorname{Cov}_{(i, j)}(z, \text { region })= \\
& \left(\vec{t}_{(k(i))}(z, \text { region })-\vec{t}_{\text {avg }(i)}(z, \text { region })\right) \\
& \sum_{k=1}^{N} \frac{\left(\vec{t}_{(k(j))}(z, \text { region })-\vec{t}_{\text {avg }(j)}(z, \text { region })\right)}{N}
\end{aligned}
$$

measured position, a covariance matrix has been constructed using Eq. (3), where $k$ is an index that shows a current signal from the set of measured signals for given position and $N$ is the total number of measured signals. $\vec{t}_{k(i)}$ indicates an $i$-th component of the $k$-th signal and $\vec{t}_{\text {avg }(i)}$ denotes $i$-th component of the average signal. $\vec{X}(\delta t, z$, region) given in Eq. (4) is the difference between the vectors describing the examined and model signals with an additional term $\delta t$, which is the parameter varied when minimizing the Mahalanobis distance:

$$
\text { (4) } \vec{X}(\delta t, z \text {, region })_{(i)}=\vec{t}_{\text {model }(i)}(z \text {, region })-\vec{t}_{k(i)}-\delta t .
$$

The reconstructed hit-position is the position of most similar signal from the library with respect to measured signal (i.e., model signal for which Mahalanobis distance computed for different bisected region is minimal ${ }^{3}$ ). The time of particle interaction is determined as a relative time between the measured signal and the most similar model signal from the library. This method also provides the determination of the gamma quantum TOF given by Eq. (5):

$$
\begin{gathered}
t_{\text {firstStrip }}=\delta t_{\text {firstStrip }} \\
t_{\text {secondStrip }}=\delta t_{\text {secondStrip }} \\
\mathrm{TOF}=t_{\text {secondStrip }}-t_{\text {firstStrip }}
\end{gathered}
$$

where $\delta t_{\text {firststrip }}$ and $\delta t_{\text {secondstrip }}$ denote shifts in time obtained for two scintillators, for which the computed Mahalanobis distance defined in Eq. (2) is lowest.

\footnotetext{
${ }^{3}$ The degree of similarity is represented by the Mahalanobis distance value.
} 


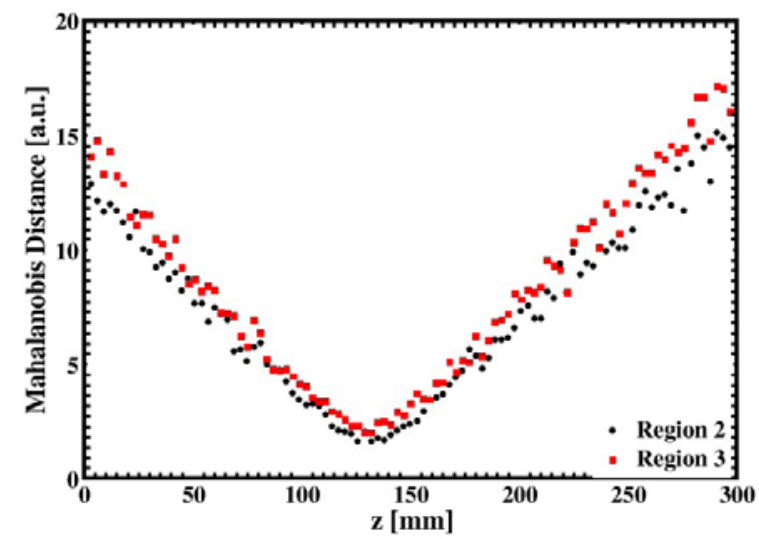

Fig. 2. Distribution of Mahalanobis distance (defined in Eq. (3)) for a signal from the central position (i.e., $150 \mathrm{~mm}$ ) from region 2 and 3.

\section{Preliminary results on performance of the method}

An example of the Mahalanobis distance distribution calculated according to Eq. (2) for one of the measured exemplary signal at central position of the strip is shown in Fig. 2. One can see a clear a)

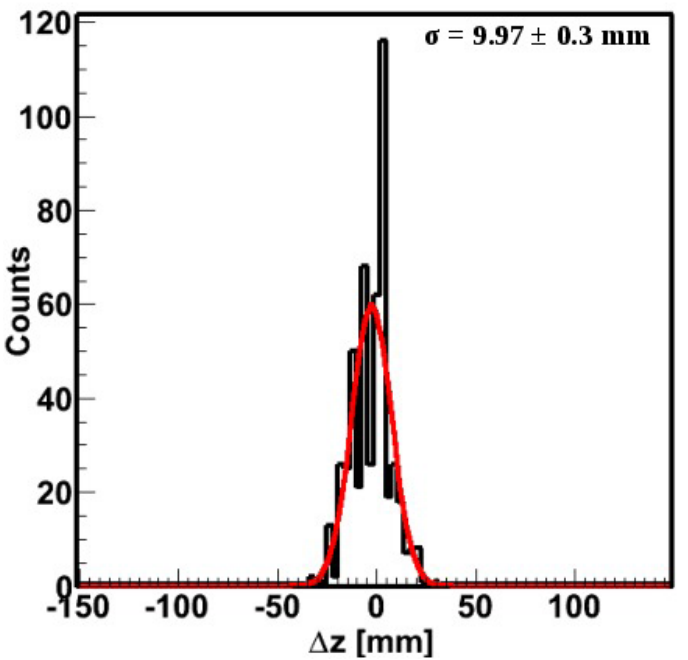

b)

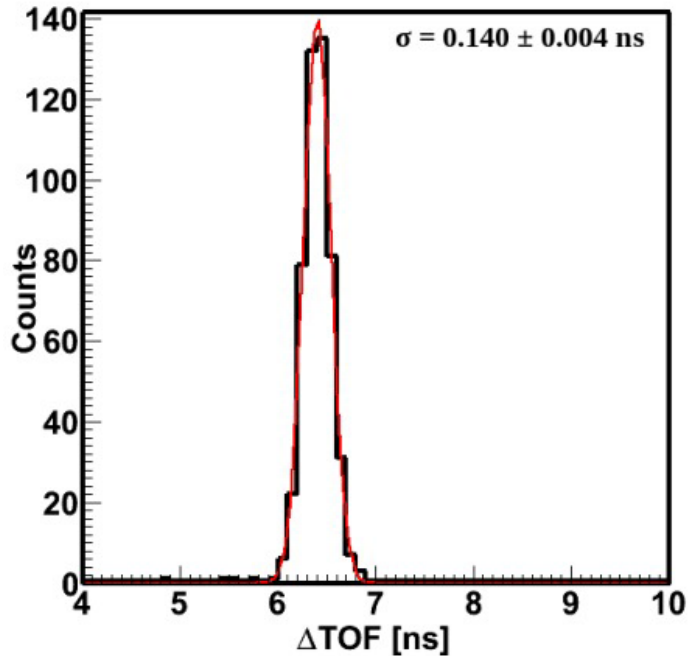

Fig. 3. (a) Distribution of differences between the true and reconstructed position $\Delta z$ for signals measured at $z=$ $150 \mathrm{~mm}$. (b) Distribution of differences between the true and reconstructed TOF for events registered at $z=150 \mathrm{~mm}$.
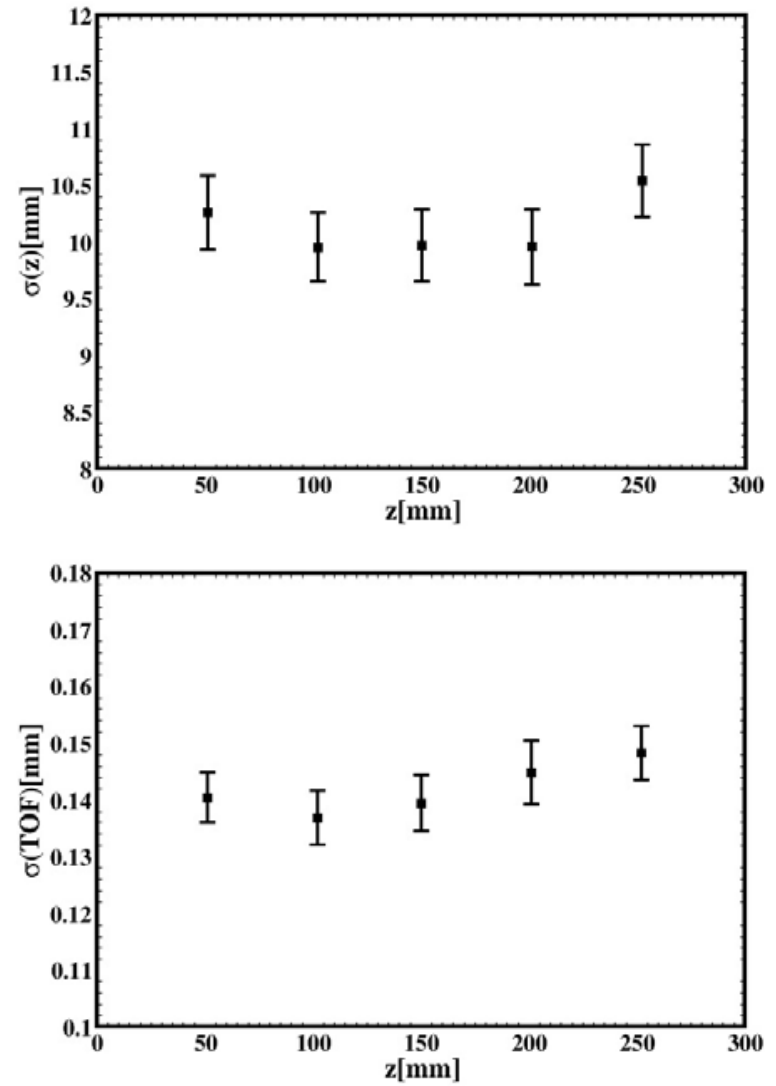

Fig. 4. (a) Position resolution as a function of the position of gamma quantum interaction. (b) TOF resolution as a function of position along the scintillator.

minimum corresponding to $z \approx 150 \mathrm{~mm}$. Figures $3 \mathrm{a}$ and $3 \mathrm{~b}$ show distributions of differences between the true and reconstructed values for position and TOF, respectively ${ }^{4}$. The estimated resolution of position and TOF reconstruction based on these distributions amounts to $\sigma_{z} \approx 10 \mathrm{~mm}$ and $\sigma_{\mathrm{TOF}} \approx 140 \mathrm{ps}$, respectively. They were determined for signals measured at several positions along the scintillator as it is shown in Figs. $4 \mathrm{a}$ and $4 \mathrm{~b}$. These results indicate that the resolutions do not change much with position.

\section{Summary}

The reconstruction method introduced in this article was validated for two-threshold levels, and the preliminary results show that it is possible to obtain a spatial resolution of about $10 \mathrm{~mm}(\sigma)$ for the gamma quanta hit position and TOF resolution of about $140 \mathrm{ps}(\sigma)$. In the present version of the J-PET, which is being built now, we install electronics that will allow us to determine time at four-threshold levels, so a further improvement is expected in the future by including more bisected regions and more threshold levels.

\footnotetext{
${ }^{4}$ In principle, the true value of TOF should be equal to zero when the source was positioned in the middle between detection modules. However, because of different electronics offsets, the reconstructed mean values of TOF may be different from zero.
} 


\section{References}

1. Moskal, P. (2014). UE Patents Nos. EP2454612B1 and EP2454611B8. Munich: European Patent Office; Moskal, P. (2014). Polish Patents Nos. WO2011008119 and WO2011008118. Kraków: Urząd Patentowy Rzeczypospolitej Polskiej.

2. Moskal, P., Salabura, P., Silarski, M., Smyrski, J., Zdebik, J., \& Zieliński, M. (2011). Novel detector systems for the Positron Emission Tomography. Bio-Algorithms and Med-Systems, 7, 73-78. [arXiv:1305.5187].

3. Moskal, P., Bednarski, T., Białas, P., Ciszewska, M., Czerwiński, E., Heczko, A., Kajetanowicz, M., Kapłon, Ł., Kochanowski, A., Konopka-Cupiał, G., Korcyl, G., Krzemień, W., Łojek, K., Majewski, J., Migdał, W., Molenda, M., Niedzwiecki, Sz., Pałka, M., Rudy, Z., Salabura, P., Silarski, M., Słomski, A., Smyrski, J., Zdebik, J., \& Zieliński, M. (2012). STRIP-PET: a novel detector concept for the TOF-PET scanner. Nucl. Med. Rev., 15, C68-C69. [arXiv:1305.5562].

4. Moskal, P., Bednarski, T., Białas, P., Ciszewska, M., Czerwiński, E., Heczko, A., Kajetanowicz, M., Kapłon, Ł., Kochanowski, A., Konopka-Cupiał, G., Korcyl, G., Krzemień, W., Łojek, K., Majewski, J., Migdał, W., Molenda, M., Niedzwiecki, Sz., Pałka, M., Rudy, Z., Salabura, P., Silarski, M., Słomski, A., Smyrski, J., Zdebik, J., \& Zieliński, M. (2012). TOF-PET detector concept based on organic scintillators. Nucl. Med. Rev., 15, C81-C84. [arXiv:1305.5559].

5. Moskal, P., Niedźwiecki, Sz., Bednarski, T., Czerwiński, E., Kapłon, Ł., Kubicz, E., Moskal, I., Pawlik-Niedźwiecka, M., Sharma, N. G., Silarski, M., Zieliński, M., Zoń, N., Białas, P., Gajos, A., Kochanowski, A., Korcyl, G., Kowal, J., Kowalski, P., Kozik, T., Krzemień, W., Molenda, M., Pałka, M., Raczyński, L., Rudy, Z., Salabura, P., Słomski, A., Smyrski, J., Strzelecki, A., Wieczorek, A., \& Wiślicki, W. (2014). Test of a single module of the J-PET scanner based on plastic scintillators. Nucl. Instrum. Methods Phys. Res. Sect. A-Accel. Spectrom. Dect. Assoc. Equip., 764, 317-321. DOI: 10.1016/j.nima.2014.07.052. [arXiv:1407.7395].

6. Kowalski, P., Moskal, P., Wiślicki, W., Raczyński, L., Bednarski, T., Białas, P., Bułka, J., Czerwiński, E., Gajos, A., Gruntowski, A., Kamińska, D., Kapłon, Ł., Kochanowski, A., Korcyl, G., Kowal, J., Kozik, T., Krzemień, W., Kubicz, E., Niedźwiecki, Sz., Pałka, M., Rudy, Z., Salabura, P., Sharma, N. G., Silarski, M., Słomski, A., Smyrski, J., Strzelecki, A., Wieczorek, A., Wochlik, I., Zieliński, M., \& Zoń, N. (2015). Multiple scattering and accidental coincidences in the J-PET detector simulated using GATE package. Acta Phys. Pol. A, 127(5), 1505-1512. DOI: 10.12693/ APhysPolA.127.1505. [arXiv:1502.04532].

7. Krzemień, W., Gajos, A., Gruntowski, A., Stoła, K., Trybek, D., Bednarski, T., Bialas, P., Czerwiński, E., Kaminska, D., Kapłon, Ł., Kochanowski, A., Korcyl, G., Kowal, J., Kowalski, P., Kozik, T., Kubicz, E., Moskal, P., Niedźwiecki, Sz., Pałka, M., Raczyński, L., Rudy, Z., Salabura, P., Sharma, N. G., Silarski, M., Słomski, A., Smyrski, J., Strzelecki, A., Wieczorek, A., Wiślicki, W., Zieliński, M., \& Zon, N. (2015). Analysis framework for the J-PET scanner. Acta Phys. Pol. A, 127(5), 1491-1494. DOI: 10.12693/ APhysPolA.127.1491. [arXiv:1503.00465].

8. Wieczorek, A., Moskal, P., Niedźwiecki, Sz., Bednarski, T., Białas, P., Czerwiński, E., Danel, A., Gajos, A., Gruntowski, A., Kamińska, D., Kapłon, Ł., Kochanowski, A., Korcyl, G., Kowal, J., Kowalski, P., Kozik,
T., Krzemień, W., Kubicz, E., Molenda, M., Pałka, M., Raczyński, L., Rudy, Z., Rundel, O., Salabura, P., Sharma, N. G., Silarski, M., Słomski, A., Smyrski, J., Strzelecki, A., Uchacz, T., Wiślicki, W., Zieliński, M., \& Zoń, N. (2015). A pilot study of the novel J-PET plastic scintillator with 2-(4-styrylphenyl)benzoxazole as a wavelength shifter. Acta Phys. Pol. A, 127(5), 1487-1490. DOI: 10.12693/APhysPolA.127.1487. [arXiv:1502.02901].

9. Białas, P., Kowal, J., Strzelecki, A., Bednarski, T. Czerwiński, E., Gajos, A., Kamińska, D., Kapłon, Ł., Kochanowski, A., Korcyl, G., Kowalski, P., Kozik, T., Krzemień, W., Kubicz, E., Moskal, P., Niedźwiecki, Sz., Pałka, M., Raczyński, L., Rudy, Z., Rundel, O., Salabura, P., Sharma, N. G., Silarski, M., Słomski, A., Smyrski, J., Wieczorek, A., Wiślicki, W., Zieliński, M., \& Zoń, N. (2015). GPU accelerated image reconstruction in a two-strip J-PET tomograph. Acta Phys, Pol. A, 127(5), 1500-1504. DOI: 10.12693/ APhysPolA.127.1500. [arXiv:1502.07478].

10. Moses, W. W., \& Derenzo, S. E. (1999). Prospects for time-of-flight PET using LSO scintillator. IEEE Trans. Nucl. Sci., 46, 474-478. DOI: 10.1109/23.775565.

11. Moskal, P., Zoń, N., Bednarski, T., Białas, P., Czerwiński, E., Gajos, A., Kamińska, D., Kapłon, Ł., Kochanowski, A., Korcyl, G., Kowal, J., Kowalski, P., Kozik, T., Krzemień, W., Kubicz, E., Niedźwiecki, Sz., Pałka, M., Raczyński, L., Rudy, Z., Rundel, O., Salabura, P., Sharma, N. G., Silarski, M., Słomski, A., Smyrski, J., Strzelecki, A., Wieczorek, A., Wiślicki, W., \& Zieliński, M. (2015). A novel method for the line-of-response and time-of-flight reconstruction in TOF-PET detectors based on a library of synchronized model signals. Nucl. Instrum. Methods Phys. Res. Sect. A-Accel. Spectrom. Dect. Assoc. Equip., 775, 54-62. DOI: 10.1016/j.nima.2014.12.005. [arXiv:1412.6963].

12. Moskal, P., Sharma, N. G., Silarski, M., Bednarski, T., Białas, P., Bulka, J., Czerwiński, E., Gajos, A., Kamińska, D., Kapłon, Ł., Kochanowski, A., Korcyl, G., Kowal, J., Kowalski, P., Kozik, T., Krzemień, W., Kubicz, E., Niedźwiecki, Sz., Pałka, M., Raczyński, L., Rudy, Z., Rundel, O., Salabura, P., Słomski, A., Smyrski, J., Strzelecki, A., Wieczorek, A., Wiślicki, W., Wochlik, I., Zieliński, M., \& Zoń, N. (2015). Hit time and hit position reconstruction in the J-PET detector based on a library of averaged model signals. Acta Phys. Pol. A, 127(5), 1495-1499. DOI: 10.12693/ APhysPolA.127.149. [arXiv:1502.07886].

13. Pałka, M., Bednarski, T., Białas, P., Czerwiński, E., Kapłon, Ł., Kochanowski, A., Korcyl, G., Kowal, J., Kowalski, P., Kozik, T., Krzemień, W., Molenda, M., Moskal, P., Niedźwiecki, Sz., Pawlik, M., Raczyński, L., Rudy, Z., Salabura, P., Sharma, N. G., Silarski, M., Słomski, A., Smyrski, J., Strzelecki, A., Wiślicki, W., Zieliński, M., \& Zoń, N. (2014). A novel method based solely on FPGA units enabling measurement of time and charge of analog signals in Positron Emission Tomography. Bio-Algorithms and Med-Systems, 10(1), 41-45. DOI: 10.1515/bams-2013-0104. [arXiv:1311.6127].

14. Raczyński, L., Moskal, P., Kowalski, P., Wiślicki, W., Bednarski, T., Białas, P., Czerwiński, E., Kapłon, Ł., Kochanowski, A., Korcyl, G., Kowal, J., Kozik, T., Krzemień, W., Kubicz, E., Molenda, M., Moskal, I., Niedźwiecki, Sz., Pałka, M., Pawlik-Niedźwiecka, M., Rudy, Z., Salabura, P., Sharma, N. G., Silarski, M., Słomski, A., Smyrski, J., Strzelecki, A., Wieczorek, A., Zieliński, M., \& Zoń, N. (2014). Novel method for hit-position reconstruction using voltage signals 
in plastic scintillators and its application to Positron Emission Tomography. Nucl. Instrum. Methods Phys. Res. Sect. A-Accel. Spectrom. Dect. Assoc. Equip., 764, 186-192. DOI: 10.1016/j.nima.2014.07.032. [arXiv:1311.6127].

15. Raczyński, L., Moskal, P., Kowalski, P., Wiślicki, W., Bednarski, T., Białas, P., Czerwiński, E., Gajos, A., Kapłon, Ł., Kochanowski, A., Korcyl, G., Kowal, J., Kozik, T., Krzemień, W., Kubicz, E., Niedźwiecki, Sz., Pałka, M., Rudy, Z., Rundel, O., Salabura, P., Sharma, N. G., Silarski, M., Słomski, A., Smyrski, J., Strzelecki, A., Wieczorek, A., Zieliński, M., \& Zoń, N. (2015). Compressive sensing of signals generated in plastic scintillators in a novel J-PET instrument. Nucl. Instrum. Methods Phys. Res. Sect. A-Accel. Spectrom. Dect. Assoc. Equip., 786, 105-112. DOI: 10.1016/j.nima.2015.03.032. [arXiv:1503.05188].

16. Mahalanobis, P. C. (1936). On the generalised distance in statistics. Proceedings of the National Institute of Sciences of India, 2(1), 49-55.

17. Bettinardi, V., Presotto, L., Rapisarda, E., Picchio, M., Gianolli, L., \& Gilardi, M. C. (2011). Physical performance of the new hybrid PET/CT Discovery-690. Med. Phys., 38(10), 5394-5411. DOI: 10.1118/1.3635220. 